\title{
The Differences Levels of RANTES and PF4 Based on the Storage of Platelet Concentrate
}

\author{
Ni Made Rindra Hermawathi, Betty Agustina Tambunan, Arifoel Hajat \\ Department of Clinical Pathology, Faculty of Medicine, Airlangga University/Dr. Soetomo Hospital, Surabaya, Indonesia. E-mail: \\ herma_md@yahoo.com
}

\begin{abstract}
Blood component transfusion is often used as the primary therapy as it is still considered safe. Platelet Concentrate (PC) transfusion plays a critical role in preventing bleeding in patients with severe thrombocytopenia. Allergic reactions are the most frequent transfusion reactions after PC administration. Regulated on Activation Normal T-Cell Expressed and Secreted (RANTES) and Platelet Factor 4 (PF4) cytokines released by platelets during PC storage are responsible for allergic reactions after transfusion. The purpose of this study was to analyze changes in RANTES and PF4 levels during PC storage. This study was an observational analytical research with a time series design carried out at the Clinical Pathology Laboratory and Blood Bank of the Dr. Soetomo Hospital, Surabaya, from June to July 2019. RANTES and PF4 levels in 27 bags derived from Platelet Rich Plasma (PRP) on storage for day 1 , day 3 , and day five were measured using the ELISA sandwich method. Subject same variant test or Friedman test was used for statistical analysis. The results showed no significant differences in RANTES and PF4 levels based on the storage duration of PCs on days 1,3 , and 5 , with $p=0.717$, and $p=0.614$, respectively. There was no difference in the storage of PCs from day 1 to day five, and there was no effect on allergic reactions after PC transfusion.
\end{abstract}

Keywords: Regulated upon activation, normal T-cell expressed and presumably secreted, platelet factor 4, platelet concentrate

\section{INTRODUCTION}

Blood and its products play an essential role in health services. Blood transfusion serves as one of the health attempts. In the context of healing diseases and health recovery, the availability of blood or its components that are sufficient, safe, easily accessible, and affordable by the public is urgently needed. ${ }^{1}$

Platelets are one of the most frequently transfused blood components. Platelet transfusion was pioneered by Murphy and Gardner and was begun to be routinely used in the 1970s. Higher reaction rates can accompany. ${ }^{2}$ Platelet transfusions compared to fresh frozen plasma and red blood cell transfusion. The risk of allergic reactions worldwide is $0.09 \%-21 \%$ in patients receiving platelet transfusion. Allergic reactions can be triggered by the presence of cytokines that accumulate in Platelet Concentrate (PC) during the storage process. ${ }^{3}$

The process of collecting, processing, and storing platelets in PC affects the structure and function of platelets. Platelet changes during the collection, processing, and storage of PC components before transfusion are defined as Platelet Storage Lesion
(PSL). ${ }^{4}$ These lesions have an impact on platelet viability and hemostasis function. In addition, platelet storage lesion is associated with morphological changes and platelet activation followed by microvesiculation and loss of platelet function, which causes platelet transfusion failure. ${ }^{5}$ Platelet storage influences platelet chemokine/ cytokine release. Therefore, this active biological material is often used as an indirect indicator of PSL.

Various types of chemokines/cytokines capable of modulating cellular receptors have been identified in platelet concentrates and are considered the cause of transfusion reactions. ${ }^{6}$ One of these chemokines is Regulated upon Activation, Normal T-cell Expressed and Presumably Secreted (RANTES) and Platelet Factor 4 (PF4) stored in a granule platelets, which will be released during platelet activation. RANTES is a chemoattractant from monocytes and has a chemoattractant effect from several subtypes of T-cells. RANTES and PF4 stimulate the release of histamine from basophils. In addition, platelet factor 4 causes exocytosis of secondary granules from neutrophils. Increased levels of RANTES and PF4 were found in PC supernatants during the storage process. ${ }^{3}$ The 
release of RANTES and PF4 chemokines from platelet granules as PSL during PC storage plays a vital role in allergic reactions after transfusion, which is the basis of this study. Research on the measurement of cytokines in the PC component is currently minimal.

This study aimed to analyze changes in RANTES and PF4 levels on day 1 , day 3 , and day 5 of the PC storage period.

\section{METHODS}

This study was an observational analytical research with a time series design conducted at the Department of Clinical Pathology and Blood Bank of the Dr. Soetomo Hospital, Surabaya. Samples were PC obtained from samples that were produced and stored at room temperature $\left(20-24^{\circ} \mathrm{C}\right)$ with agitation that met the sample inclusion and exclusion criteria. The inclusion criteria were PC samples produced during the study period, which were processed following the Minister of Health regulation number 91 of 2015 on storage days 1, 3, 5. The exclusion criteria were defective PC and the PC component without swirling, that the viability of the platelets was doubtful. Samples were taken by consecutive sampling technique from March 2019 until September 2019.

This study was conducted on 27 PC samples produced during the study period at the Blood Bank of Dr. Soetomo Hospital following Minister of Health Regulation Number 912015 on storage of day 1 , day 3 , and day 5 . The PC component with color changes and without any swirling were excluded from this study.

This research had received approval from the Health Research Ethics Committee of the Dr. Soetomo Hospital, Surabaya, with number 1261/KEPK/VI/2019.

Platelet concentrate stored on day one at a temperature of $20-24^{\circ} \mathrm{C}$ with the agitation was collected in plain tubes as much as $3 \mathrm{~mL}$ utilizing a connecting tube in the PC bag, which would be clamped first and cut-off at the ends of the connecting tube, a total of $3 \mathrm{~mL}$ sample was then collected in plain tubes. The PC bag connecting pipe was closed again using a heat sealer. The plain tube containing $3 \mathrm{~mL}$ PC was centrifuged at 4,000 $\mathrm{g}$ for 15 minutes at $20-24^{\circ} \mathrm{C}$. The supernatant was removed and placed in one $\mathrm{mL}$ aliquots. The process of collecting PC in plain tubes and transferring supernatant to aliquots was carried out in a biosafety cabinet to prevent bacterial contamination. Platelet concentrate components taken for re-sampling were stored at $20-24^{\circ} \mathrm{C}$ with agitation. The same procedure was carried out to collect PC samples with a storage period of days three and day 5 . The aliquots were stored in a refrigerator at $-80^{\circ} \mathrm{C}$ until 27 samples were collected. Storage in the fridge with a temperature of $-80^{\circ} \mathrm{C}$ will maintain the stability of RANTES and PF4 levels in less than three months. RANTES and PF4 measurements were carried out by an ELISA sandwich method based on the Elabscience ${ }^{\circledR}$ insert kits made from United States. ${ }^{7.8}$

The same subject variant analysis was used for RANTES and PF4 data with normal distribution, while Friedman analysis was used for data without normal distribution, $p<0.05$ was significant.

\section{RESULTS AND DISCUSSIONS}

The quality assurance of the RANTES and PF4 test in this study was carried out before sample measurement. Quality assurance was performed by checking the expiration date, lot number of each kit, and reagent storage according to the instructions listed on the equipment. Quality assurance was also carried out by performing a control. The control results showed that both the low level and high level were included in the value of the control range.

The sample size used in this study was 27 samples. Sampling was conducted in June-July 2019 at the Blood Bank of the Dr. Soetomo Hospital. Characteristics of the samples as donor blood groups can be seen in Table 1.

Table 1. Characteristics of samples

\begin{tabular}{ccc}
\hline $\begin{array}{c}\text { Blood } \\
\text { Grouping }\end{array}$ & $\begin{array}{c}\text { Platelet Concentrate } \\
\text { (bags) }\end{array}$ & $\begin{array}{c}\text { Percentage } \\
\text { (\%) }\end{array}$ \\
\hline$A+$ & 4 & 14.8 \\
$B+$ & 11 & 40.7 \\
$O+$ & 9 & 33.3 \\
$A B+$ & 3 & 11.1 \\
Total & 27 & 100 \\
\hline
\end{tabular}

A total of 27 bags of PC blood component were used in this study, and the most obtained PC bags were $B+$ blood type as many as 11 bags (40.7\%) and the least with $A B$ blood type as many as three bags (11.1\%) such as seen in Table 1 . This finding was because the consecutive sampling technique was adjusted to the availability of PC reserves in the Blood Bank of Dr. Soetomo Hospital.

Shapiro-Wilk test results in Table 2 showed that all platelet data had normal distribution ( $p>0.05$ ). 
Therefore, platelet counts between observations were determined using analysis of the same variant of subjects because all platelet count data were normally distributed. The normality test results of RANTES data showed the abnormal distribution of data $(p<0.05)$; therefore, the Friedman test was used to determine the differences in RANTES levels between observations. The normality test of the PF4 level also showed abnormally distributed data $(p<0.05)$; therefore, the Friedman test was used to determine the difference in PF4 levels between observations.

The results of differential platelet counts also showed the characteristics of the samples on day 1 , day three, and day five and were analyzed using the same variant analysis of the subjects as shown in Table 2.

The results of the analysis of variance with the same subjects showed no significant difference in platelet counts between observations ( $p>0.05)$, and it indicated that platelet counts on day 1 , day 3 , and day 5 were homogenous. Homogeneity of the samples in this study was also indicated by the Coefficient of Variation (CV) between observations as less than $30 \%$ (Table 3 ).

The differential test levels of RANTES day 1 , day 3 , and day 5 used Friedman test are illustrated in Table 4. Friedman test results showed no significant differences in RANTES levels between observations $(p>0.05)$.

The boxplot diagram of the RANTES level in Figure 1 showed almost the same median at the time of observation on day 1 , day 3 , and day 5 but did not show any significant difference. Two samples of RANTES on day 1 were outlier data, and one sample was extreme data, whereas one sample with extreme values was found on day 3. Three samples had high RANTES levels in this study, which were observed on first-day storage, and one sample had high RANTES levels on day three storage, which was also high on day one storage. This result was caused by the high number of leukocytes donors in the PC bag, but no high number of leukocytes in the three samples with high RANTES levels. This high level of RANTES might

Table 2. Normality test results

\begin{tabular}{lccc}
\hline & Platelet Concentrate (bags) & p-value & Normality \\
\hline Platelet count & 27 & 0.091 & Normal \\
Day 1 & 27 & 0.226 & Normal \\
Day 3 & 27 & 0.378 & Normal \\
Day 5 & & \\
RATES & 27 & $<0.001$ & Not-normal \\
Day 1 & 27 & $<0.001$ & Not-normal \\
Day 3 & 27 & 0.001 & Not-normal \\
Day 5 & & & \\
PF4 & 27 & $<0.001$ & Not-normal \\
Day 1 & 27 & 0.339 & Normal \\
Day 3 & 27 & $<0.001$ & Not-normal \\
Day 5 & & & \\
\hline
\end{tabular}

Table 3. Platelet count differential test

\begin{tabular}{cccc}
\hline Platelet Count & $\begin{array}{c}\text { Platelet Concentrate } \\
\text { (bags) }\end{array}$ & $\begin{array}{c}\text { Mean } \pm \text { Standard Deviation } \\
\left(\mathbf{x ~ 1 0 ^ { 3 }} \mathbf{/ \mathbf { m m } ^ { 3 }}\right)\end{array}$ & p-value \\
\hline Day 1 & 27 & $690,70 \pm 184,32$ & \\
Day 3 & 27 & $701,74 \pm 185,28$ & 0.188 \\
Day 5 & 27 & $689,07 \pm 204,65$ & \\
\hline
\end{tabular}

Table 4. Results of differential levels of RANTES

\begin{tabular}{cccc}
\hline Levels of RANTES & Platelet Concentrate (bags) & Median $(\mathbf{m i n}-\mathbf{m a x})(\mathbf{p g} / \mathbf{m L})$ & p-value \\
\hline Day 1 & 27 & $104.24(9.54-813.97)$ & \\
Day 3 & 27 & $105.79(9.32-610.18)$ & 0.717 \\
Day 5 & 27 & $98.94(12.72-531.20)$ & \\
\hline
\end{tabular}




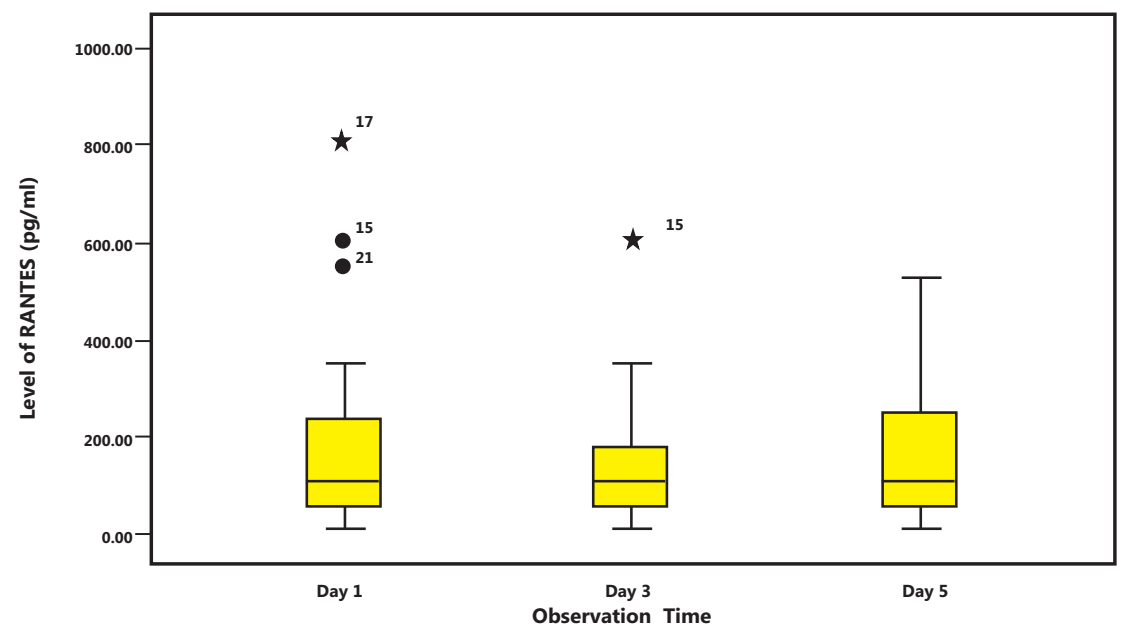

Figure 1. Boxplot diagram of RANTES level to the observation time

Table 5. Result of PF4 differential test

\begin{tabular}{cccc}
\hline Levels of PF4 & Platelet Concentrate (bags) & Median $(\mathbf{m i n}-\mathbf{m a x}) \mathbf{( n g} / \mathbf{m L})$ & p-value \\
\hline Day 1 & 27 & $35.18(15.44-113.84)$ & \\
Day 3 & 27 & $30.87(12.43-55.13)$ & 0.641 \\
Day 5 & 27 & $30.58(14.89-91.11)$ & \\
\hline
\end{tabular}

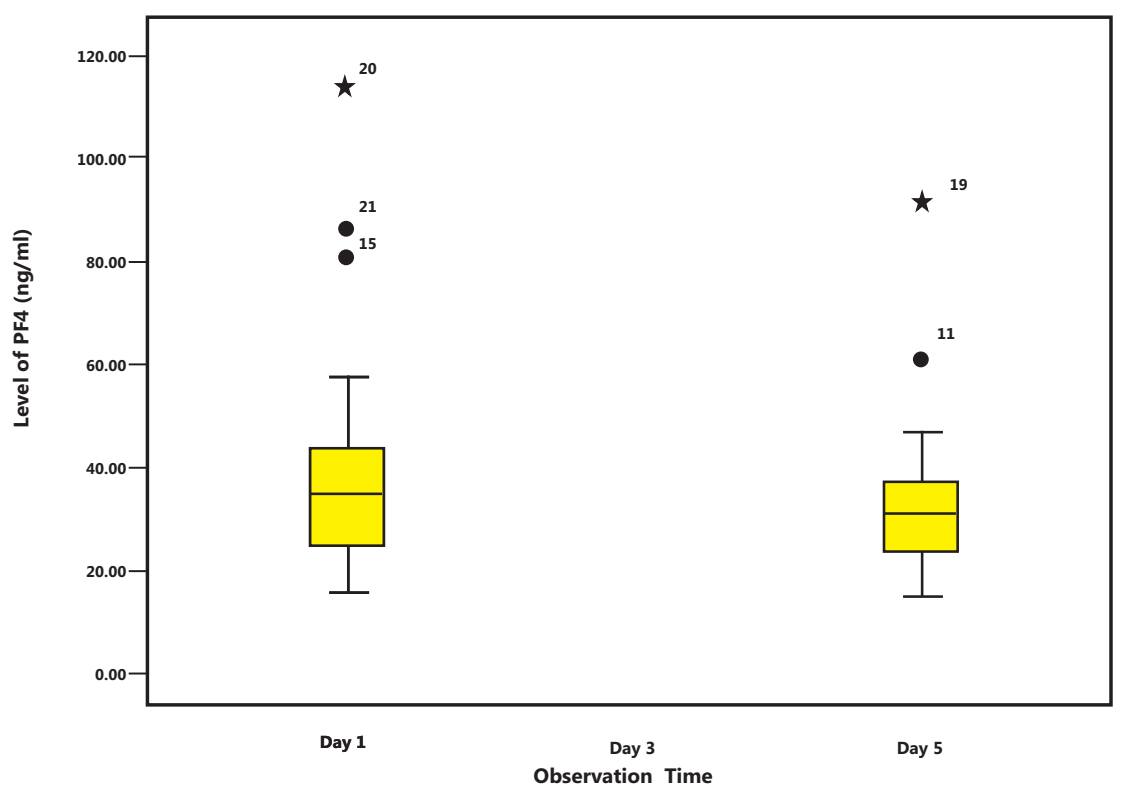

Figure 2. Boxplot Diagram of PF4 levels to the observation time

be caused by other factors that could not be explained in this study. The increase of RANTES levels during PC storage could also be caused by bacterial contamination of PC components suggested being the main cause of the formation of PSL from warehouse of PC.

The Friedman test results in Table 5 showed no significant difference in PF4 levels between observations $(p>0.05)$.

The boxplot diagram of the PF4 levels seen in Figure 2 showed almost the same median on the third observation time on day 1 , day 3 , and 5 but did not show any significant differences. Three samples showed PF4 levels outside the minimum to maximum range in this study, and two samples showed extreme values, far above the median PF4 levels obtained in the results of this study.

Some results of RANTES and PF4 levels measurement in this study showed high or extreme values that it was necessary to repeat the measures that had high or extreme values. The results of this study were the same as previous studies conducted 
by Wadhwa et al., suggesting that RANTES and PF4 were detected in the PC starting on the first day of storage. The research undertaken by Wadhwa et al. was different from this study because it used PC derived from apheresis with two other techniques. In contrast, this study used a PC derived from Platelet Rich Plasma (PRP). This study showed that RANTES and PF4 levels were not significantly different during the storage period of day 1 , day 3 , and day 5 . In contrast, the study by Wadhwa showed significant differences during the storage period of PC on day one and day 4/5. RANTES and PF4, which are mainly stored in the cytoplasm of platelet a granules, will be removed during $P C$ 's processing and storage period. Increased levels of RANTES in the supernatant indicate platelet activation or destruction. ${ }^{10}$

Another study conducted by Bubel et al. showed that RANTES and PF4 were excreted in PC and accumulated during the PC storage period. The study conducted by Bubel compared PC derived from apheresis with PC derived from buffy coat and showed no difference in the RANTES content in the supernatant in these two methods. This result could be caused by half-life, different concentrations of platelet a granules and other mechanisms of chemokines release caused by platelet activation. ${ }^{11}$

Research conducted by Tormey showed that the release of cytokines and chemokines occurred in the early stages of PC production. The stages that cause the highest increase in RANTES release happened in the initial stages of PC production from PRP. The expenditure of RANTES in large amounts occurred due to the use of high-speed spin (hard spin) when producing PC. ${ }^{13}$ This can support the results of this study that RANTES was obtained from the first day of PC storage, and no significant differences were found in RANTES levels over PC storage time. ${ }^{12}$

This study showed that RANTES and PF4 chemokines were detected from the first day of PC storage, which indicated the activation of platelets but did not increase in number, and the levels of RANTES and PF4 did not differ significantly during the storage period. This finding showed no increase in inactivation or destruction of platelets during the PC storage period on day 1 , day 3 to day 5 . Therefore, storage of the blood components at the Blood Bank of the Dr. Soetomo Hospital has followed the requirements stated in the Regulation of the Minister of Health of the Republic of Indonesia Number 91 of 2015 concerning blood transfusion service standards. Quality testing of PC blood components was also performed every month to maintain the quality of blood products released from the Blood
Bank of Dr. Soetomo Hospital. Data of the allergic reactions after administration of PC blood components during 2018 showed 15 cases of allergic reactions from 29,679 bags of PC delivered. Allergic reactions as transfusion reactions are probably caused by other biological response modifiers factors such as complement fragments, cytokines, kininogen, and histamine that were not examined in this study.

\section{CONCLUSIONS AND SUGGESTIONS}

The RANTES and PF4 chemokines in the PC blood component were not different during the $\mathrm{PC}$ storage period on days 1,3 , and 5 . However, some results of RANTES and PF4 levels measurement in this study showed high or extreme values required to repeat the tests. Therefore, further research was needed to compare RANTES and PF4 levels in PC blood components made with a different method.

\section{ACKNOWLEDGMENT}

Researchers would like to thank the Blood Transfusion Installation and the Blood Bank of the Dr. Soetomo Hospital, which has permitted to carry out this research to be successfully completed. It was expected that this research could be helpful and be used to assess the quality of the PC at the Blood Bank of the Dr. Soetomo Hospital, Surabaya.

\section{REFERENCES}

1. Kemenkes RI. Peraturan Menteri Kesehatan Republik Indonesia nomor 91 tahun 2015 tentang standar pelayanan tranfusi darah. 2016; 6-7.

2. Forest SK, Hod EA. Management of the platelet refractory patient. Hematology/Oncology Clinics of North America, 2016; 30(3): 665-77.

3. Sut $C$, Tariket $S$, Aubron $C$. The non-hemostatic aspect of transfused platelets. Frontiers in Medicine, 2018; 5: 1-11.

4. Ahmed A, Leheta O, Younes S. In-vitro assessment of platelet storage lesion in leukoreduced random donor platelet concentrate. Blood Transfusion, 2010; 8(1): 28-35.

5. Armenia D, Tambunan BA. Evaluation of storage length to blood component platelet concentrate quality in the Blood Bank, Dr. Soetomo General Hospital Surabaya Indonesia. Indian Journal of Forensic Medicine \& Toxicology, 2020; 14(4): 908-913.

6. Seghatchian J. Platelet storage lesion: An update on the impact of various leukoreduction processes on the biological response modifiers. Transfusion and Apheresis Science, 2006; 34(1): 125-30.

7. Elabscience. Manual book-human RANTES 
(Regulated on Activation, Normal T-Cell Expressed and Secreted) ELISA kit. 2017; 1-11.

8. Elabscience. Manual book-human PF4 (Platelet Factor 4) ELISA kit. 2017; 1-11.

9. Shrivastava M. The platelet storage lesion. Transfusion and Apheresis Science, 2009; 41(2): 105-13.

10. Wadhwa $M$, Seghatchian MJ, Dilger $P$, Sands $D$, Krailadisiri $\mathrm{P}$, et al. Cytokines in WBC-reduced apheresis PCs during storage: A comparison of two WBC-reduction methods. Transfusion, 2000; 40(9):
1118-26.

11. Shukla R, Patel T, Gupte S. Release of cytokines in stored whole blood and red cell concentrate: Effect of leukoreduction. Asian Journal of Transfusion Science, 2015; 9(2): 145-149.

12. Tormey C, Stack G. Use of a cytokine-release assay to demonstrate loss of platelet secretory capacity during blood bank processing and storage. Archives of Pathology \& Laboratory Medicine, 2014; 138(11): 1481-7. 